\title{
Practice improves performance on a coronary anastomosis simulator, attending surgeon supervision does not
}

\author{
Jennifer Walker, MD
}

See related article on pages 12-7.

First, I congratulate the authors for a well-executed study in an area of great interest in the general and cardiothoracic surgical training arena. Deliberate practice is the underlying theme in the simulation world, and there is no question that practice improves performance. The questions that remain at large are how much the innate skill, intrinsic motivation, clinical experience, and competitive environment influence the performance of the trainee.

The optimal scenario is still a balance between teaching and learning in simulation, but I think we all agree that putting more of the responsibility on the learner is more effective. The authors show that no practice with minimal previous exposure yields a minor but measurable improvement. On the contrary, the conclusion that supervised practice yields measurable but less improvement than unsupervised practice has many interpretations.

The unsupervised learner has the full burden of improvement and has to be self-critical and diligent with the hope that the next critique will validate his or her practice and skill. The supervised learner intrinsically relies on cues from the teacher to guide the learning. For basic techniques such as the one described, this is a valid observation.
Unfortunately, most of cardiac surgery and more advanced general surgery require feedback for complex tasks that have become the standard for achieving the appropriate outcomes. Cardiac surgical patients continue to have more comorbidities and require more advanced decisionmaking and anticipation of complications. Leaving the learning of these advanced tasks to the untrained learner is not fair or productive. Protocols for simulating complex adverse scenarios are in the process of being developed and validated.

This article is a useful exercise to demonstrate the importance of motivating young learners. Simulation training not only engages the learner but also improves interest in surgical specialties and more advanced simulation training.

The entire cardiothoracic community understands the importance of deliberate practice for the appropriate training of our future cardiothoracic surgeons. The ideal use of low-and high-fidelity simulators, one-on-one versus video- or peer-based learning, and the most effective way to evaluate performance and progress still remain a work in progress.

Again, I congratulate the authors on this study, which I am sure will be a launchpad for more research and observation.

From the Massachusetts General Hospital, Boston, Mass.

Disclosures: Author has nothing to disclose with regard to commercial support.

Received for publication Sept 17, 2014; accepted for publication Sept 18, 2014.

Address for reprints: Jennifer Walker, MD, Massachusetts General Hospital, 55 Fruit

St, EDR 105, Boston, MA 02114 (E-mail: jdwalker@ partners.org).

J Thorac Cardiovasc Surg 2015;149:18

$0022-5223 / \$ 36.00$

Copyright $₫ 2015$ Published by Elsevier Inc. on behalf of The American Association

for Thoracic Surgery

http://dx.doi.org/10.1016/j.jtcvs.2014.09.058 